\title{
Primer año y medio de una consulta de enfermedad renal crónica avanzada
}

\author{
M $^{\text {a }}$ Auxiliadora Santana Díez, $\mathbf{M}^{\mathrm{a}}$ Rosario Aguirremota Corbera, Marta González Vélez, M $^{\mathrm{a}}$ Pilar Lodeiro \\ Herraiz
}

Hospital Universitario Cruces. Bizkaia

\section{Introducción:}

Diferentes estudios demuestran que al aumentar la información que un paciente posee sobre su patología mejora su calidad de vida, aumenta la adherencia al tratamiento y retrasa el progreso de su enfermedad. En Mayo del 2011, se creó nuestra consulta de Enfermedad Renal Crónica Avanzada (E.R.C.A.), para proporcionar una atención más integral a los pacientes que acuden a las consultas médicas de insuficiencia renal avanzada, pendientes de entrar en programa sustitutivo (P.E.P.). Con anterioridad se creó un registro acorde a la ley, en nomenclatura NANDA-NOC-NIC sobre dos diagnósticos enfermeros influyentes en su calidad de vida: "Déficit de conocimientos" y "Temor" para poder sistematizar y evaluar mejor los cuidados. La realidad actual de nuestra consulta no se ciñe a la idea inicial, pacientes de P.E.P., pues tenemos pacientes que nos llegan remitidos de otras procedencias de nefrología, por ello nos planteamos como objetivos de este trabajo:

- Ver el perfil de la población que estamos atendiendo.

- Comprobar si es nuestra gráfica de registro un instrumento eficaz para la consulta E.R.C.A.

\section{Metodología:}

\section{Diseño:}

Descriptivo retrospectivo observacional desde el comienzo de la consulta hasta el 31 de Enero del 2013.

\section{Población:}

Todos aquellos pacientes que fueron remitidos a nuestra consulta. Para saber cuál es el perfil de nuestra población nos fijamos en las siguientes variables: edad, sexo, procedencia, $\mathrm{n}^{\circ}$ de consultas, $\mathrm{n}^{\circ}$ de intervenciones en cada NIC concreto y evolución en la consulta 1,3 y 5 del indicador correspondiente al NIC más demandado. Se utilizaron dos registros formato Excel, un listado de los pacientes a los que otorgamos un código para preservar su identidad y partiendo de este listado otro con los datos de las diferentes variables.

\section{Resultados:}

Desde Mayo del 2011 hasta final de Enero del 2013, han sido atendidos 50 pacientes, su edad media es de 63,7 años, siendo el $62 \%$ varones. Procedentes un $46 \%$ de consulta E.R.C.A., un $38 \%$ de consulta de trasplante renal, un $14 \%$ de la sala de hemodiálisis y un $2 \%$ de diálisis peritoneal. La media de consultas fue de 4,28 , siendo la intervención más requerida la de "Enseñanza dieta prescrita". Sobre esta intervención quisimos evaluar, gracias a los indicadores, si alcanzábamos el NOC u objetivo que planteamos en el origen. En el proceso de recogida de datos se vio que un número significativo de pacientes repetían el indicador en sucesivas consultas y no avanzaban. Se entrevistó a las enfermeras que atendieron la consulta en ese periodo y se observó que se producía una interpretación de los parámetros del protocolo de valoración, lo que hacía que fueran poco objetivos.

\section{Conclusiones:}

La consulta atiende a un grupo de pacientes más amplio del diseño original. La gráfica se ajusta bastante bien a nuestras necesidades. Hay que ser más estrictos a la hora de aplicar el protocolo para no dejar espacio a la subjetividad que hace inviable la 
utilización de los datos. Para ello se creó una comisión interna que estableció criterios consensuados en base a su experiencia y a la nueva realidad de la consulta.

\section{Referencias Bibliográficas}

1. Álvarez R, Velasco S. La consulta de prediálisis de enfermería: logros y oportunidades de mejora. Rev Soc Esp Enferm Nefrol. 2007; 10(3):6-11.

2. Barrios R, González IM, Canca JC, Barea MA, Salas M, Bravo MJ, et al. ¡Estamos en obras! Construyendo un futuro en equipo: Consulta IRCA. En: Libro en DVD de comunicaciones del XXXII Congreso de la Sociedad Española de Enfermería Nefrológica; Cádiz del 3-6 de Octubre del 2007. HOSPAL; 2007. P 31-35.

3. Estalella M, Martínez L. Manual de buenas prácticas para la Creación de Bases de Datos de Investigación. UCICEC Hospital de Cruces (CAIBER). 2009.
4. Llaguno $E$, Herrero $M$, Arteche $C$, Gurrutxaga $A$, Barrueco P, García de Diego A, et al. Programa educacional y de seguimiento de pacientes con insuficiencia renal avanzada. En: Libro en DVD de comunicaciones del XXIX Congreso de la Sociedad Española de Enfermería Nefrológica; Tenerife del 2-5 de Octubre del 2004. HOSPAL; 2004. P 273274.

5. Pulido F, Arribas E, Pulido JF, González F, Aragonzillo I. Tres años en la consulta ERCA. En: Libro en DVD de comunicaciones del XXXIV Congreso de la Sociedad Española de Enfermería Nefrológica; Pamplona. HOSPAL; 2009. P 250-251.

6. Pulido F, Arribas P, Fernández A. Nivel de satisfacción de los pacientes en la consulta de enfermería (ERCA). En: Libro en DVD de comunicaciones del XXXII Congreso de la Sociedad Española de Enfermería Nefrológica; Cádiz del 3-6 de Octubre del 2007. HOSPAL; 2007. P 93.

7. Salamanca AB. El aeiou de la investigación en enfermería. Madrid. Fuden; 2013. 